\title{
Sclerosing mesenteritis mimicking mesenteric malignancy
}

\author{
Jung Yeop Lee ${ }^{1}$, Ki Jung Yun ${ }^{2}$, Dong Baek Kang ${ }^{3}$, Tae Hyeon Kim ${ }^{1}$, and Hyung Ku Chon ${ }^{1}$
}

Departments of ${ }^{1}$ Internal Medicine, ${ }^{2}$ Pathology, and ${ }^{3}$ Surgery, Wonkwang University Hospital, Iksan, Korea

Received: July 4, 2017

Revised : October 8, 2017

Accepted: October 8, 2017

\section{Correspondence to}

Hyung Ku Chon, M.D.

Tel: +82-63-859-2564

Fax: +82-63-855-2025

E-mail: gipb2592@wku.ac.kr
A 68-year-old man who had undergone radical prostatectomy for prostate cancer 5 years ago presented to our hospital complaining of an $8 \mathrm{~kg}$ weight loss within 2 months and a palpable periumbilical mass. A carbohydrate antigen 19-9 was noted to be increased to $85 \mathrm{U} /$ $\mathrm{mL}$, but other laboratory studies were unremarkable. An abdominal computed tomography scan showed a large spiculated mass with focal calcifications and surrounding inflammation along the small bowel mesentery (Fig. $1 \mathrm{~A}$ and 1B). Positron emission tomography revealed an intense hypermetabolic mesenteric lesion (maximum standardized uptake value $=6.4$ ) (Fig. 1C). An exploratory laparotomy was performed to obtain a differential diagnosis, especially to distinguish between a mesenteric malignancy and other benign conditions. Meticulous adhesiolysis and small bowel segmental resection with end-to-end anastomosis was performed. The gross specimen showed a yellowish hard mass forming fat infiltration along mesentery (Fig. 2A). Microscopically, the mass showed fat necrosis, sclerosing fibrosis, and focal infiltration of chronic inflammatory cells with fibroblasts without a granuloma, vasculitis, or malignancy (Fig. 2B). Based on histopathological results, he was diagnosed with sclerosing mesenteritis (SM), and following relief of symptoms he was discharged 10 days later, without any complications.

SM includes a spectrum of inflammatory disorders involving the adipose tissue of the bowel mesentery without known etiology. Clinical manifestations are nonspecific, and there are no
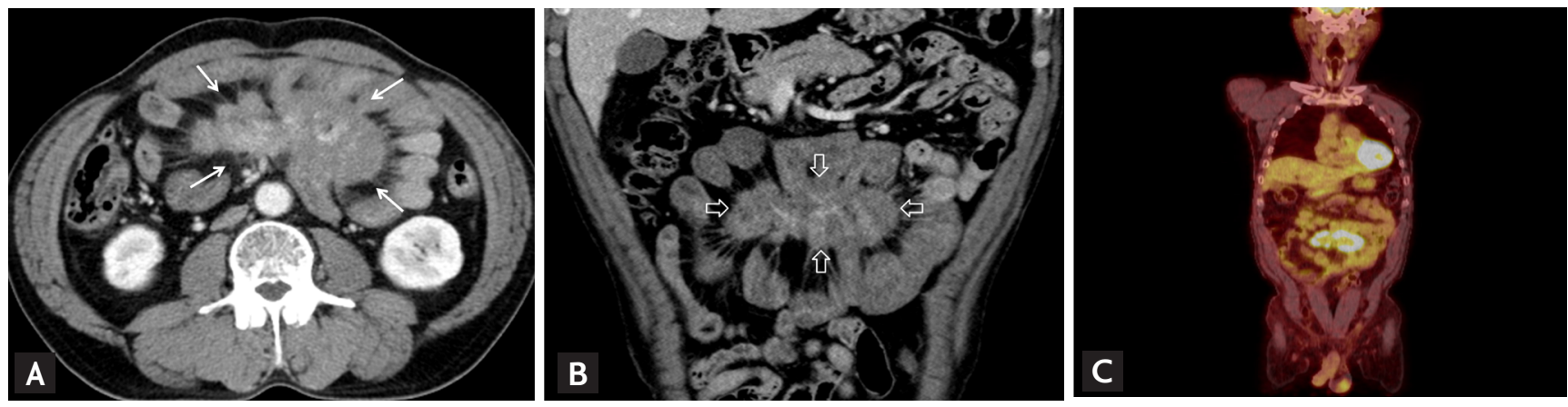

Figure 1. (A) Axial contrast-enhanced computed tomography (CT) image showing an 11-cm spiculated mass (between arrows) demonstrating mild enhancement and focal tumor calcifications in the mesentery. (B) Coronal CT of the abdomen also reveals a large confluent mass (between open arrows) attached to the loops of the small bowel. (C) A large lobulated mesenteric mass with increased fluoro-2-deoxy-D-glucose uptake (maximum standardized uptake value $=6.4$ ) is identified on positron emission tomography images. 

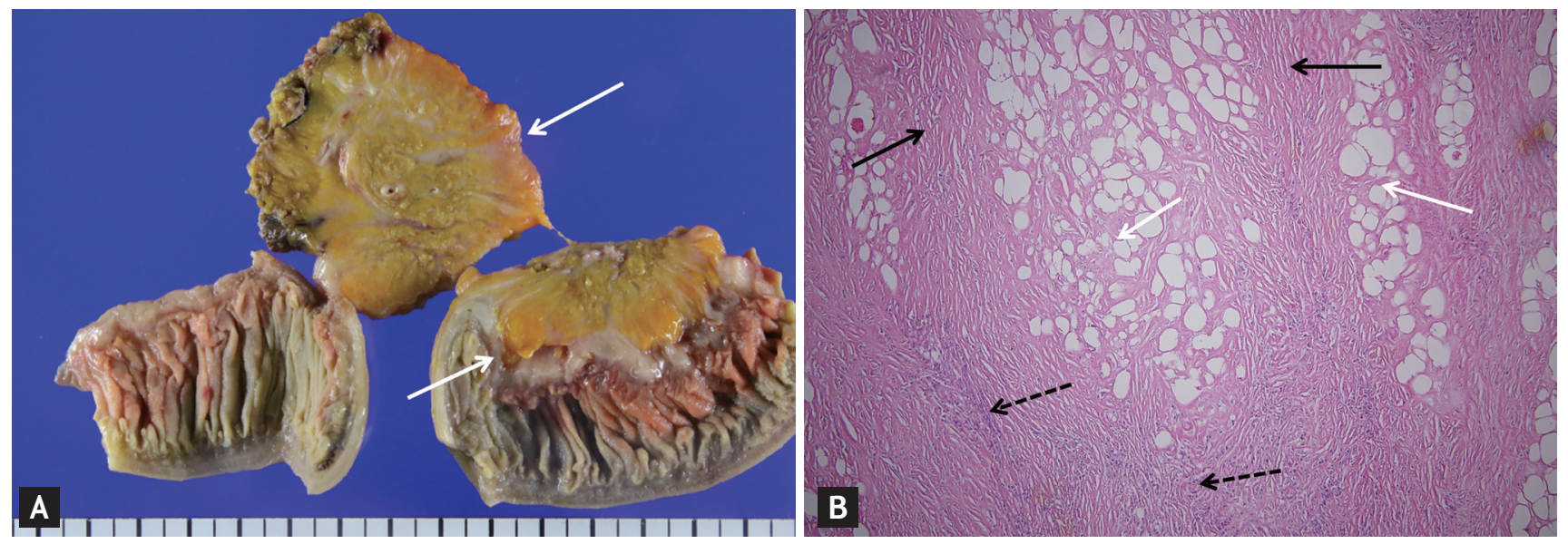

Figure 2. (A) Gross surgical specimen shows a yellowish ill-defined hard lesion with a fibrotic cut surface and congestion (white arrows). The lesion extends into the intestinal subserosa.(B) Microscopic findings reveal fat necrosis (white arrows), sclerosing fibrosis with dense collagen (black arrows), and clusters of inflammatory cells (black dotted arrows) in the mesenteric mass $(\mathrm{H} \& \mathrm{E}, \times 100)$.

pathognomonic findings observed using cross-sectional imaging. Thus, diagnosing SM preoperatively may be difficult, and a mass typically associated with SM might mimic a primary or secondary mesenteric malignancy, especially in patients with a history of malignancy, elevated tumor markers, and/or severe weight loss as was seen in our patient. We propose that SM may be considered among the differential diagnosis in patients presenting with a mesenteric mass, even in patients with a history of malignancy.
Written informed consent was obtained from the patient who participated in this study.

\section{Conflict of interest}

No potential conflict of interest relevant to this article was reported.

\section{Acknowledgments}

This paper was supported by Wonkwang University in 2018. 